\title{
Un posible análisis sobre el control público del servicio del agua en Buenos Aires y Santa Fe, Argentina
}

\section{Analysis on a possible public water service control in Buenos Aires and Santa Fe, Argentina}

\section{RESUMEN}

El presente artículo analiza las funciones de los organismos vinculados con el servicio del agua en Buenos Aires y Santa Fe, Argentina, a los fines de detectar posibles superposiciones de funciones que podrían generar una ineficiencia en el servicio.

Desde el punto de vista metodológico se utilizó el método de observación documental que consiste en obtener información mediante la percepción selectiva, ilustrada e interpretativa de un fenómeno determinado. Asimismo se utilizaron los métodos analítico y comparativo de datos, siendo que se comparó legislación de distintas jurisdicciones de Argentina, y se aplicó la teoría de indicadores diseñada por Global Integrity para evaluar el rendimiento de los organismos de control del servicio del agua.

Luego de analizarse todas las obligaciones que surgen de la normativa de estos organismos y la información suministrada en las redes conceptuales, debe destacarse que existen áreas de solapamiento para el ámbito de la Provincia de Buenos Aires, en donde se generan situaciones dudosas respecto a qué organismo acudir ya que varios poseen la misma facultad para el mismo ámbito territorial.

Lo anterior, permitió concluir que el aspecto más problemático que se ha analizado se vincula con el funcionamiento de los organismos de control, a través del ejercicio de su poder de policía. Se afirma entonces, que la existencia de un alto nivel de cumplimiento legal por parte de las empresas de agua, va a depender directamente de los siguientes ámbitos:

a) Funcionamiento de los organismos de control.

b) La calidad de la normativa vigente.

\section{PALABRAS CLAVE}

Control público, servicio de agua, solapamientos legales. 


\section{ABSTRACT}

This article examines the roles of the agencies linked with the water in Buenos Aires and Santa Fe, Argentina, for the purpose of detecting possible overlapping of functions that could generate an inefficiency in the service. From the methodological point of view was used the method of documentary observation that consists of obtaining information through selective, interpretative and enlightened perception of a given phenomenon.

After analyzing all the obligations arising from the regulations of these bodies and the information provided in the conceptual networks, it should note that there are areas of overlap in the area of the province of Buenos Aires, in where it generated dubious situations about which agency go since several have the same Faculty,, for the same territory.

The above, concluded that the most problematic aspect that was scanned is linked with the operation of the supervisory bodies, through the exercise of its police power. Then, says that the existence of a high level of compliance by water companies will depend directly on the following areas:

(a) Functioning of the supervisory bodies.

(b) The quality of legislation

\section{KEYWORDS:}

Public control, water service, legal overlaps.

\section{INTRODUCCIÓN}

Desde los años ochenta, las reformas efectuadas en el sector del servicio del agua en Argentina han pasado de un modelo institucional centralizado a un esquema municipal numeroso, fragmentado y muy heterogéneo. En este sentido, "el argumento central fue la necesidad de acercar la solución de los problemas de la población, con el fin de aprovechar las iniciativas locales y la proximidad a los usuarios." (Fernández; Jouravlev; Lentini; Yurquina, 2009, p.6).

El supuesto que subyace a este modo es que, la gestión de los servicios llevada adelante por las unidades estatales más pequeñas, posibilitaría una gestión más adecuada que la desarrollada por las unidades estatales centrales. La descentralización apunta al desarrollo de procesos de gestión de los servicios que permitan un mayor acceso de la población de menores recursos y atenuar las desigualdades territoriales o regionales (Mutuberría, 2007).

En tal sentido, una de las transformaciones institucionales del sector que se han producido en la década de los noventa en Argentina, ha sido la separación de los roles de fijación de políticas, regulación, control y prestación de los servicios públicos. El Estado se reservó las funciones de formulación de las políticas sectoriales, la regulación y el control de las concesiones, delegando la prestación en empresas privadas. Uno de los puntos claves es que el Estado debe participar mediante el control del cumplimiento de las normas pautadas en el contrato de concesión (plan de inversiones, tarifas y servicios en general) (Ehrhardt; Groom; Halpern; O'Connor, 2007).

Todo lo anterior justificó la creación (aunque tardía porque ya las empresas concesionarias se encontraban funcionando) de los denominados Entes Reguladores ${ }^{1}$. Diversos autores, como Rouse (2007) consideran que un organismo es independiente cuando tiene la libertad de fijar tarifas, actuar con fuerza determinante en la toma de decisiones, imponer sanciones, hablar con los medios, e informar sobre cualquier aspecto en

1.- Esto tuvo lugar en el mes de Febrero de 2007, por lo que la empresa de agua estuvo casi un año sin contar con el control de este organismo. 
relación con las funciones regulatorias.

En relación con los aspectos que se analizarán, otros autores como Solanes (2009, p.2) afirman que "el funcionamiento de los entes de control es fundamental porque la calidad de la normativa dictada por cada Estado determina las condiciones del servicio, de los precios y de los impactos ambientales". La problemática de la organización se basa en la programación y el ordenamiento de un conjunto de funciones, de modo optimo en relación con los objetivos fijados y para esto se proponen organigramas ideales donde se deberán describir: i). Cargos, ii). Normas; iii). Procedimientos, y; iv). Planes.

El principal objetivo del presente artículo, es analizar el control existente sobre el gobierno y sobre las empresas que brindan el servicio de agua, a través de la utilización del sistema de medición implementado por una de las categorías de indicadores de Global Integrity.

\section{Metodología}

\section{a) Fase exploratoria:}

En virtud de lo anterior, se recopiló normativa nacional vinculada con el sistema de control del servicio de agua en Buenos Aires y en Santa Fe (Argentina). Además se realizó una selección de obras doctrinarias y de aplicación empírica sobre los mecanismos y organismos de control del servicio del agua. A tal fin se utilizaron los siguientes buscadores jurídicos: Microjuris, Ecolex y Lexpro.

b) Fase descriptiva:

1- La información recogida en la etapa anterior fue clasificada y categorizada (utilizando los criterios de división territorial, jurisdiccional y doctrinario), a fin de facilitar su análisis en una etapa posterior.

2- Se realizó una descripción de "casos" en los que el núcleo duro de la cuestión fue el sistema de control del servicio del agua. De ello se obtuvo información relevante para arribar a algunas conclusiones generales. c) Fase analítica:

1- El análisis de los documentos vinculantes y no vinculantes recogidos en la fase exploratoria tuvo por objeto detectar los principios y marcos legales que, debido a su reiteración constante y uniforme, fueron tomados en cuenta para enriquecer el marco teórico de esta investigación.

2- Se categorizaron los estudios de casos publicados en revistas o por organismos nacionales e internacionales de reconocida trayectoria.

Se utilizó el método de observación documental que consiste en obtener información mediante la percepción selectiva, ilustrada e interpretativa de un fenómeno determinado. Dentro de las posibles modalidades se implementó la "observación directa", ya que los datos se recogieron directamente de los fenómenos percibidos mediante registros sistematizados con la recolección.

De igual manera, se utilizaron los métodos analítico y comparativo de datos, siendo que se comparó legislación de distintas jurisdicciones de Argentina, y se aplicó la teoría de indicadores diseñada por Global Integrity para evaluar el rendimiento de los organismos de control del servicio del agua.

\section{LAS PRIVATIZACIONES $Y$ NACIONALIZACIONES DEL SERVICIO DEL AGUA EN ARGENTINA}

En Argentina, se produjeron las primeras privatizaciones del servicio del agua potable y de saneamiento del mundo, y además al mismo tiempo se contaba con una extensa historia institucional y legal. Desde el año 1800 hasta 1980 la empresa estatal Obras Sanitarias de la Nación fue la responsable de suministrar los servicios de agua y alcantarillado a las principales ciudades de Argentina, mientras que las más pequeñas estaban a cargo de las provincias y de los municipios.

En 1949 mediante el dictado de la Ley 13.577 se creó la Administración General de Obras Sanitarias de la Nación (OSN), que era dependiente del Ministerio de Obras Públicas de la Nación. En dicha ley se estableció que esta institución tendría las funciones 
de realizar estudios, construir, y renovar las obras de provisión de aguas y saneamiento urbano de la Capital Federal y de todas las ciudades de la Argentina. Asimismo, se le otorgó la facultad de explorar y utilizar los recursos de aguas subterráneas.

En 1980 se le transfirieron los servicios a las provincias argentinas, quedando este organismo únicamente a cargo de la gestión del agua de la ciudad de Buenos Aires y de algunos partidos de la provincia de Buenos Aires. Posteriormente, mediante los programas de reforma del Estado implementados por la Ley 23.696 de Emergencia Administrativa de 1989, se previó la privatización de una serie de servicios públicos entre los cuales se encontraba el del agua y saneamiento.

En 1992 se implementó la privatización de Obras Sanitarias de la Nación a través del Decreto 999 de $1992^{2}$, y mediante el Decreto 787 de 1993 se aprobó el contrato suscripto entre el Estado Nacional y la empresa Aguas Argentinas S.A. donde se le otorgó la concesión del servicio público de provisión de agua y desagües cloacales de Buenos Aires y de 17 partidos de la Provincia de Buenos Aires.

Luego de catorce años de concesión, el Decreto 303/2006 rescindió el contrato suscripto entre el Estado Nacional y la empresa Aguas Argentinas S.A. A través del Decreto 304/2006 se constituyó la actual sociedad que regula estos servicios públicos, y que se denomina Agua y Saneamientos Argentinos Sociedad Anónima dentro de la órbita de la Secretaría de Obras Públicas del Ministerio de Planifica- ción Federal, Inversión Pública y Servicios. Esta sociedad se creó bajo la normativa establecida por la Ley 19.550 de sociedades comerciales dictada en 1984.

Una de las características más importantes de esta empresa, es que el noventa por ciento del capital accionario le pertenece al Estado Nacional, ejerciendo su titularidad el Ministerio de Planificación Federal, Inversión Pública y Servicios (siendo esto intransferible). El diez por ciento restantes les corresponde a los ex trabajadores de OSN adheridos al programa de propiedad participada. La concesión de Santa Fe experimentó la misma evolución habiéndose transferido el servicio a la empresa Aguas Provinciales de Santa Fe S.A. en el año 1995. También en el año 2006, mediante el Decreto Provincial 243, se rescindió el contrato de concesión del servicio de agua.

\section{LAS FUNCIONES DE LOS ORGANISMOS DE CONTROL PÚBLICO DEL SERVICIO DEL AGUA EN BUENOS AIRES Y EN SANTA FE, ARGENTINA}

En los ámbitos territoriales analizados, existe una gran cantidad de organismos de control del servicio del agua, los cuales en algunos casos cumplen funciones de mucha relevancia.

A continuación se describirán las principales funciones de estos organismos para los casos de Buenos Aires y de Santa Fe, los cuales luego se interrelacionarán con los indicadores seleccionados para el presente artículo.

\section{Cuadro $\mathrm{N}^{0} 1$}

Organismos ámbito nacional y de la Ciudad de Buenos Aires

Período concesionado a empresas privadas(1993-2006)

\begin{tabular}{|l|l|}
\hline Organismo de control & Funciones principales \\
\hline Ente Tripartito de Obras de Servicios & Dicta regulación. \\
de Saneamiento (ETOSS) & Controla el cumplimiento de la concesión. \\
& Monitoreo de planes de inversión. \\
& Atención de reclamos de usuarios. \\
& Imposición de penalidades a las empresas. \\
\hline
\end{tabular}

2.- El mismo aprueba el Reglamento administrativo regulatorio de los distintos aspectos de los servicios públicos de provisión de agua potable y desagües cloacales de competencia de Obras Sanitarias de la Nación. 


\section{Cuadro $\mathrm{N}^{\circ} 2$}

Organismos ámbito nacional y de la Ciudad de Buenos Aires

Período de provisión estatal del servicio (a partir del año 2006)

\begin{tabular}{|l|l|}
\hline Organismo de control & Funciones principales \\
\hline Ente Regulador del Agua y saneamiento (ERAS) & $\begin{array}{l}\text { Fiscaliza la calidad del servicio, la protección de los } \\
\text { intereses de la comunidad yel control. }\end{array}$ \\
\hline Agencia de Planificación (APLA) & $\begin{array}{l}\text { Coordina y realiza el seguimiento de la planificación y } \\
\text { de la ejecución de las obras. } \\
\text { Realiza el seguimiento de planes para universalizar } \\
\text { el servicio de agua. Interactúa con los municipios. }\end{array}$ \\
\hline
\end{tabular}

\section{Cuadro $\mathrm{N}^{\circ} 3$}

Organismos de la Provincia de Santa Fe

Período concesionado a empresas privadas (1993-2006)

\begin{tabular}{|l|l|}
\hline Organismo de control & Funciones principales \\
\hline Ente Regulador de Servicios Sanitarios (ENRESS) & Dicta todas las reglamentaciones que sean atinentes \\
& al ejercicio de su competencia regulatoria. \\
& Aprueba y controla que los prestadores cumplan con \\
& los planes de mejoras y desarrollo. \\
& Atiende los reclamos de los usuarios. \\
& Verifica que los prestadores cumplan el régimen \\
& tarifario. \\
& Controla los prestadores en todo lo que se refiere al \\
& mantenimiento de los bienes afectados al servicio. \\
& Controla la calidad química y microbiológica y los \\
& demás parámetros de calidad del agua. \\
& Ejerce el poder disciplinario y sancionatorio. \\
& Observa el fiel cumplimiento por parte del conce- \\
& sionario de todas las obligaciones que surjan de la \\
& ley, el pliego de bases y condiciones y demás normas \\
& aplicables debiendo imponer las sanciones y \\
& correctivos que correspondan. \\
\hline
\end{tabular}

\section{Cuadro $\mathrm{N}^{\circ} 4$}

Organismos de la Provincia de Santa Fe

Período de provisión estatal del servicio (a partir del año 2006)

\begin{tabular}{|l|l|}
\hline Organismo de control & Funciones principales \\
\hline Ente Regulador de Servicios Sanitarios (ENRESS) & $\begin{array}{l}\text { Idem anterior. Se trata de una empresa mayoritaria- } \\
\text { mente con capitales públicos. }\end{array}$ \\
\hline Secretaría de Medio Ambiente (Pcia. de Santa Fe) & $\begin{array}{l}\text { Coordina y dirige un programa de control de efluentes } \\
\text { y saneamiento de cursos superficiales }\end{array}$ \\
\hline
\end{tabular}




\section{Cuadro $\mathrm{N}^{\circ} 5$}

Organismos de la Provincia de Buenos Aires

\begin{tabular}{|c|c|}
\hline Organismo & Funciones principales \\
\hline $\begin{array}{l}\text { Ministerio de Infraestructura, Vivienda } \\
\text { y Servicios Público }\end{array}$ & $\begin{array}{l}\text { Planificar y programación las obras públicas, en } \\
\text { conjunto con los municipios en que se desarrollen. } \\
\text { Analiza el dictado de normas relacionadas con la } \\
\text { contratación, construcción y conservación de las } \\
\text { obras públicas; y la intervención en la dirección, } \\
\text { organización y fiscalización del registro de empresas } \\
\text { contratistas de obras públicas y de consultoría } \\
\text { relacionadas a ellas con arreglo a la legislación } \\
\text { provincial vigente. Tiene a cargo la regulación de los } \\
\text { servicios de agua y cloacas. }\end{array}$ \\
\hline $\begin{array}{l}\text { Subsecretaria de servicios públicos. } \\
\text { Dirección Provincial de Servicios Públicos de Agua } \\
\text { y Cloacas, Ministerio de Infraestructura de la } \\
\text { Provincia de Buenos Aires }\end{array}$ & $\begin{array}{l}\text { Llevaa cabo la planificación, ejecución y supervisión } \\
\text { de los programas de obras destinadas al manteni- } \\
\text { miento, mejora y ampliación de los servicios de } \\
\text { captación, potabilización, almacenamiento, trans- } \\
\text { porte y distribución de agua potable y recepción, } \\
\text { tratamiento y disposición de desagües cloacales. } \\
\text { Realiza la administración de la información sobre la } \\
\text { problemática, la demanda y las condiciones de la } \\
\text { infraestructura, la calidad y la cobertura de los } \\
\text { servicios públicos de agua y cloacas; la ejecución de } \\
\text { la programación de los planes de abastecimiento, } \\
\text { cobertura, optimización y expansión de los servicios } \\
\text { públicos de agua y cloacas. } \\
\text { Ejecuta las obras de infraestructura para el agua y } \\
\text { cloacas mediante obras de captación, potabilización, } \\
\text { almacenamiento, transporte y distribución de agua } \\
\text { potable, tratamiento y disposición de desagües } \\
\text { cloacales. }\end{array}$ \\
\hline $\begin{array}{l}\text { Organismo Provincial de Desarrollo Sostenible } \\
\text { (OPDS) }\end{array}$ & $\begin{array}{l}\text { Autoridad de aplicación en materia ambiental en el } \\
\text { ámbito de la Provincia de Buenos Aires, como } \\
\text { entidad autárquica de derecho público. Planifica, } \\
\text { formula, proyecta, fiscaliza, ejecuta la política } \\
\text { ambiental, y preserva los recursos naturales } \\
\text { ejerciendo el poder de policía, y, fiscalizando todo } \\
\text { tipode efluentes. } \\
\text { Planifica y coordina con los organismos compe- } \\
\text { tentes, la ejecución de programas de educación y } \\
\text { de política ambiental destinada a mejorar y preservar } \\
\text { la calidad ambiental. }\end{array}$ \\
\hline
\end{tabular}


Servicio Provincial de,Agua Potable y

Saneamiento Rural (SPAR)

Autoridad del Agua (ADA)

Organismo descentralizado cuya función es ejecutar programas para poblaciones rurales.

Lleva adelante la planificación hidrológica, la reglamentación, supervisión y vigilancia de todas las actividades y obras relativas al estudio, captación, uso, conservación y evacuación del agua.

Promociona programas de educación formal e informal sobre el uso racional del agua.

Organismo de Control de Aguas de Buenos Aires Tiene a su cargo la función de controlar y fiscalizar la (OCABA), Subsecretaría de Servicios Públicos, Ministerio de Infraestructura de la Provincia de Bs. As. prestación de los servicios públicos de agua y de cloacas, la defensa de los intereses de los usuarios, la intervención en las cuestiones relacionadas con la actividad de las prestatarias del servicio; y vela por la protección del medio ambiente.

Fuente: Ministerio de Infraestructura, Vivienda y Servicios Públicos, de la Subsecretaría de Modernización del Estado, la Autoridad del Agua, el Organismo de Control de Aguas de Buenos Aires y Aguas Bonaerenses S.A.

\section{RESULTADOS}

\subsection{Aplicación de una selección de indicadores de Global Integrity}

Global Integrity es un proveedor líder internacional de información que analiza las tendencias de la corrupción y del buen gobierno en los diferentes países del mundo, y es conocido principalmente por su reporte anual sobre integridad nacional (Global Integrity Report). Este informe consiste en una compilación de estudios de casos de países (tanto cualitativos como cuantitativos), preparados por investigadores y académicos. Estos innovadores indicadores de integridad evalúan diversos cuestiones normativas e institucionales vinculadas con la apertura, la calidad del gobierno, y con la existencia de mecanismos para prevenir la corrupción en cada país.

Todos estos indicadores están reforzados por un proceso de control de calidad y anclados mediante precisos criterios de evaluación y múltiples referencias ${ }^{3}$. Luego de la descripción de las principales funciones de los organismos de control, se las vinculó y agrupó tomando como referencia a los indicadores de Global Integrity seleccionados. En este sentido consideramos que no es necesario que se creen nuevas estructuras estatales, ya que las mismas son suficientes. Esto es así porque todos los organismos contemplaron los aspectos medidos por los tres indicadores de Global Integrity.

A continuación en los siguientes gráficos se han unificado algunas de las funciones realizadas por los organismos de control de los tres ámbitos analizados (Nacional, Ciudad de Buenos Aires, Provincia de Buenos Aires y de Santa Fe). Allí se puede visualizar que existen varios organismos que realizan las mismas funciones (produciéndose una superposición), y en consecuencia generando un servicio menos eficiente. 


\section{Gráfico $N^{\circ} 1$}

Vinculación de las funciones de los organismos con uno de los indicadores prácticos de Global Integrity $\left(I^{4}\right.$

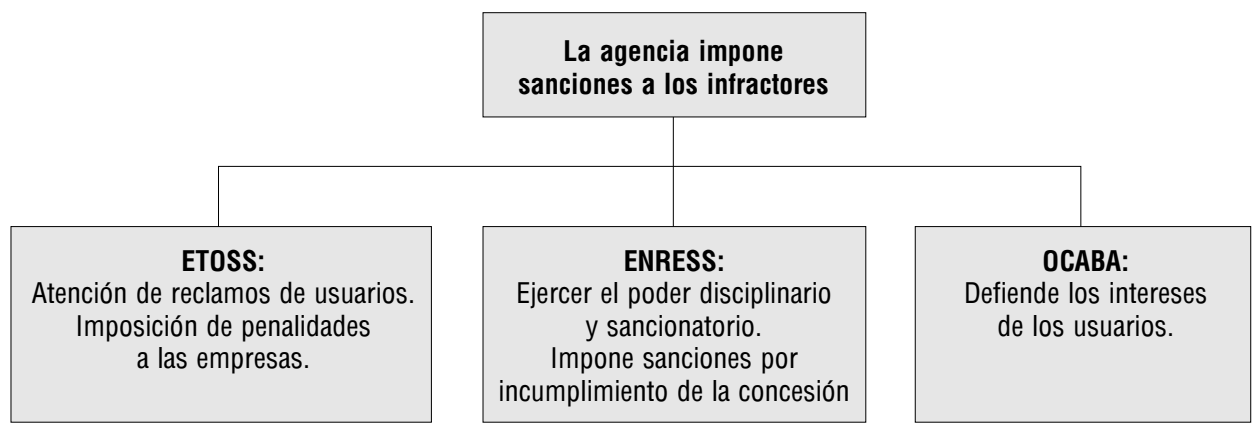

En este caso se produce una superposición de funciones entre el ETOSS y OCABA para el ámbito de la misma concesión, ya que el ENRESS pertenece al ámbito de Santa Fe

\section{Gráfico $\mathrm{N}^{\circ} 2$}

Vinculación de las funciones de los organismos con uno de los indicadores prácticos de Global Integrity (II) (dividido en dos gráficos)
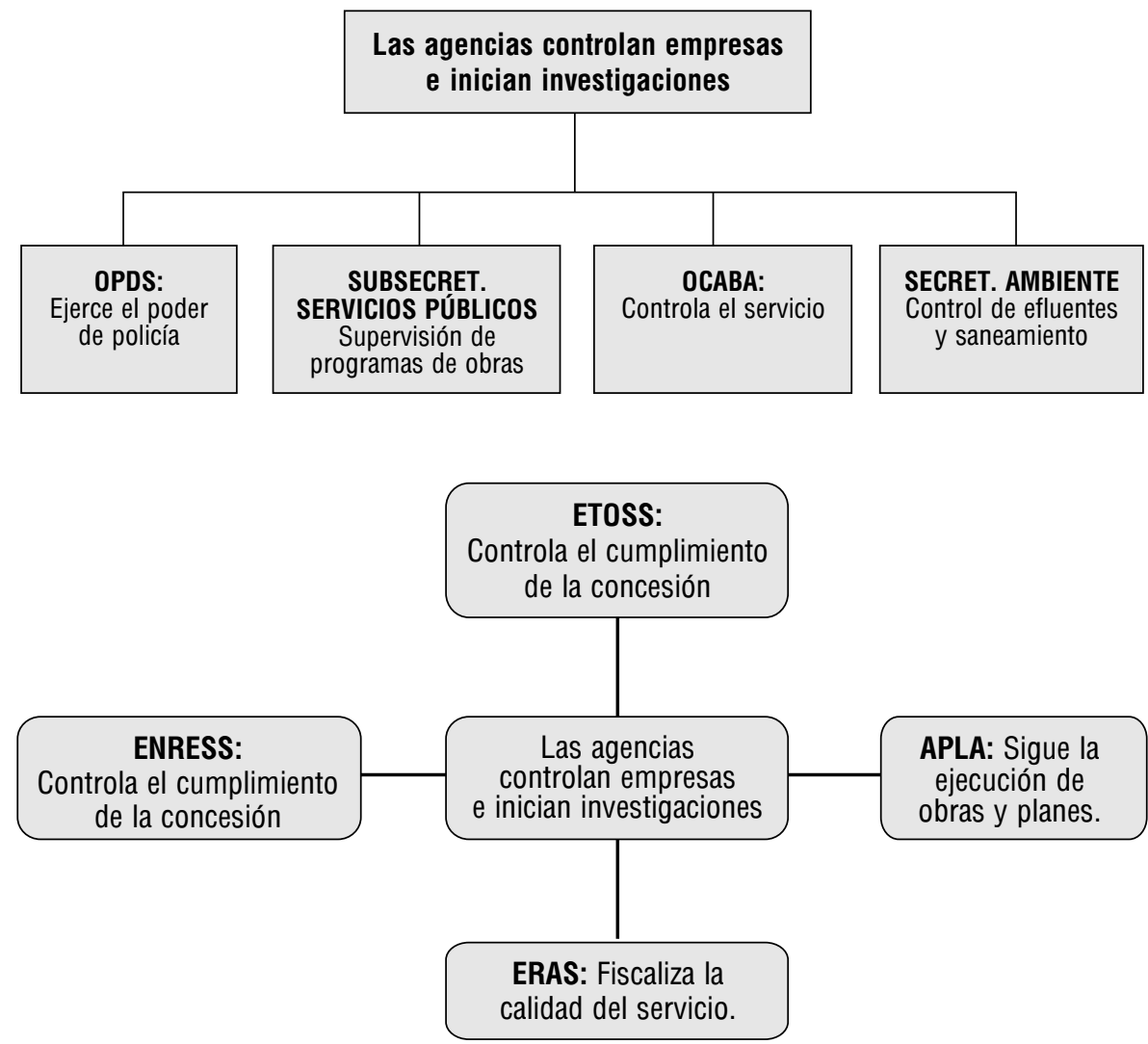

4.- Indicador Práctico de Global Integrity: En la práctica, cuando fuese necesario, la agencia, serie de agencias o mecanismo equivalente de control de las empresas públicas impone sanciones a los infractores.

5.- Indicador práctico: En la práctica, ¿la agencia, serie de agencias o mecanismo equivalente de control de las empresas públicas inicia investigaciones de forma independiente?. 
Aquí la superposición de tareas es muy amplia, ya que siete organismos diferentes se ocupan de iniciar y resolver expedientes vinculados con el control del cumplimiento normativo del servicio del agua. No se encuentran interconectados entre sí, y algunos están ubicados en diferentes ámbitos territoriales (Ciudad Autónoma de Buenos Aires y Provincia de Buenos Aires). Esto genera que existan causas paralelas que datan sobre los mismos asuntos, y en muchos casos sin que sea posible tener acceso a la información necesaria para su correcta resolución.

\section{Gráfico $\mathrm{N}^{\circ} 3$}

Vinculación de las funciones de los organismos con uno de los indicadores legales de Global Integrity ${ }^{6}$ (dividido en dos gráficos).

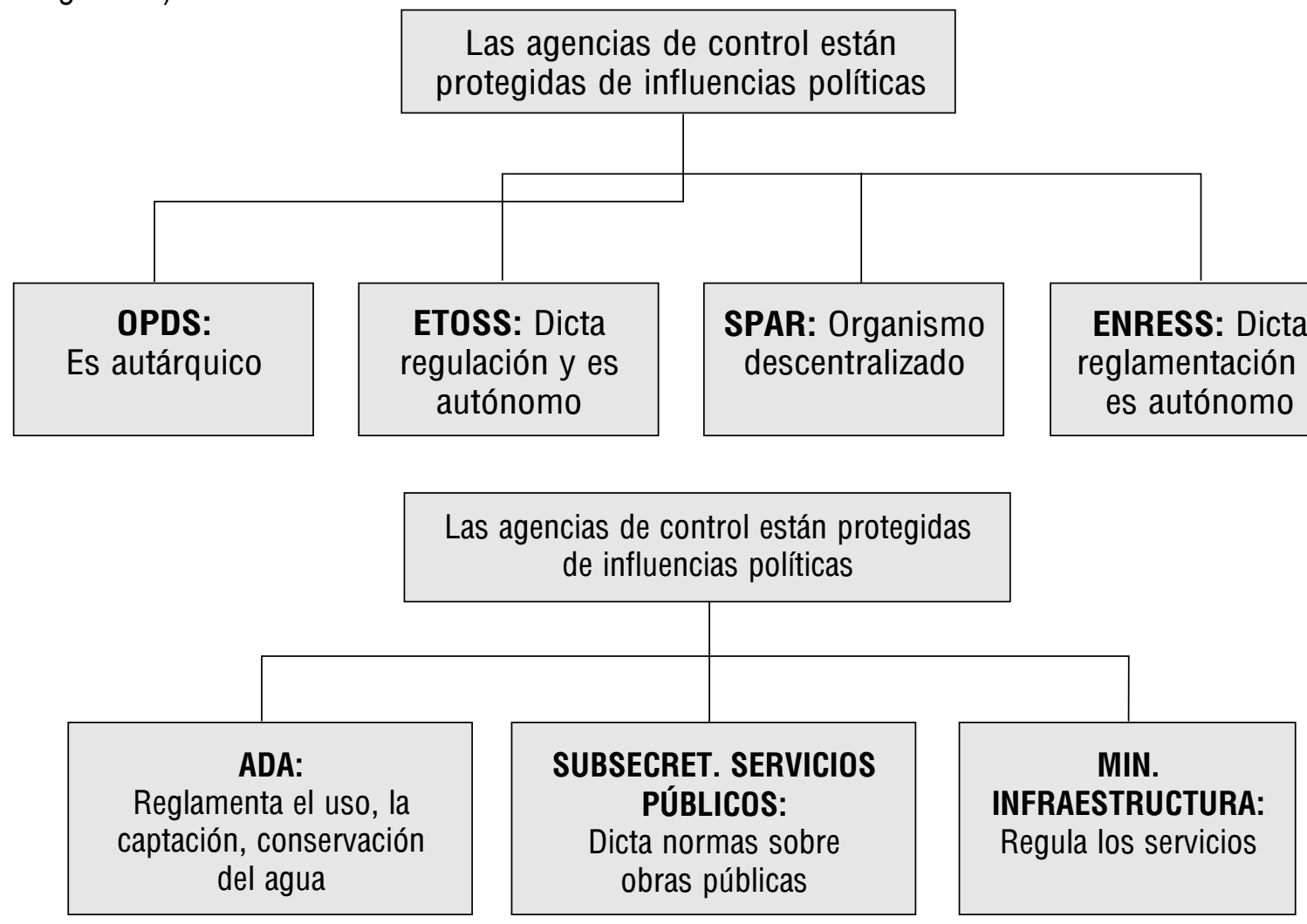

En este gráfico se muestra que la superposición es favorable a la eficiencia del servicio, ya que teóricamente estos organismos poseen independencia legal de las influencias políticas. Por eso, sería recomendable instaurar herramientas de control y sanciones por incumplimiento de las mismas.

Estos indicadores pueden medir el nivel de funcionamiento de los organismos, sosteniendo que el mismo es satisfactorio de acuerdo a los aspectos tratados por la normativa vigente.

Otro de los aspectos fundamentales que hemos advertido luego del análisis de las atribuciones legales que poseen los distintos organismos de control del servicio de agua, es la falta de separación existente entre el ámbito político y el técnico, que genera que se produzcan situaciones de ineficiencia y/o de injusticia.

6.- Indicador legal: En el Derecho vigente, ¿̇la agencia, serie de agencias o mecanismo equivalente de control de las empresas públicas se encuentra protegido de las influencias políticas?. 
Todos los aspectos anteriormente analizados, (los vinculados con la separación de funciones de los organismos de control del servicio de agua), deben estar suficiente y adecuadamente regulados. Diversos autores coinciden en que la tendencia mayoritaria dentro de la práctica del ámbito privado o público del servicio del agua, generalmente responde a orientaciones políticas ${ }^{7}$. Esto puede vislumbrarse en los casos en donde los políticos no quieren elevar las tarifas de los servicios (a pesar de no poder cubrir los costos ni poder mantener adecuadamente la infraestructura), para no perder popularidad frente a los electores, poniendo en riesgo la calidad del servicio.

Otro de los casos habituales es el de no realizar planificaciones a largo plazo en relación con las inversiones, para asegurar a los consumidores un servicio de mejor calidad. Esto muchas veces provoca que se incumpla con diversa normativa vigente, y que los organismos encargados del control no actúen con su máximo rendimiento.

Otro caso es el de las empresas proveedoras de este servicio (tanto en los períodos concesionados a las empresas privadas como en los períodos de provisión estatal), no han invertido en micromedidores para realizar un control del consumo de agua y cobrar conforme a los resultados de los mismos a los consumidores. Considero al igual que Rouse (2007) que la clave es separar legalmente a la política del área operacional, permitiendo que personal capacitado y con experiencia pueda trabajar de manera independiente sin sufrir ninguna interferencia política (p.25). En Argentina no se cuenta con ninguna normativa que regule esta temática, existiendo de esta manera un vacío legal.

\section{SOLAPAMIENTOS LEGALES EN LAS FUNCIONES DE LOS ORGANISMOS}

A continuación se destacarán gráficamente los organismos relacionados con el servicio del agua, dentro del ámbito de la concesión de Buenos Aires, respecto de los cuales se superponen sus funciones dificultando su actividad de contralor:

\section{Gráfico $\mathrm{N}^{0} 4$}

Control y fiscalización del servicio

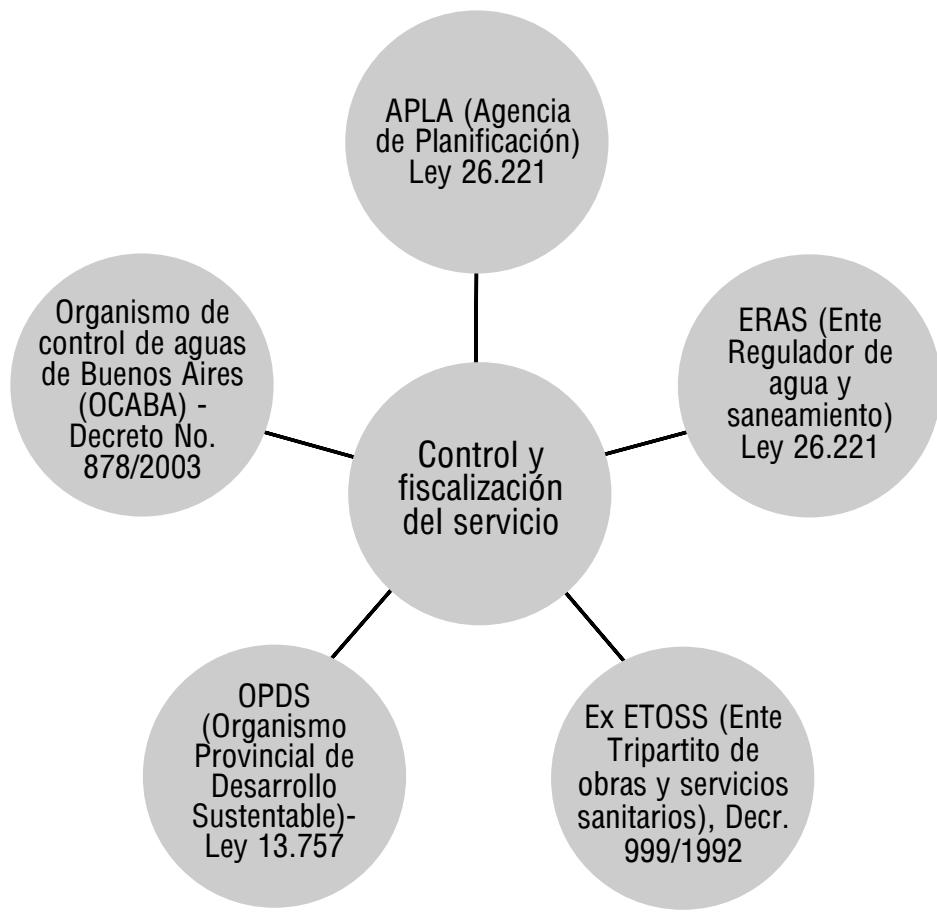

7.- Vid. Transparency International, 2008: 6; United NationsI Development, 2011: 7; Budds, J.; Mcgranahan, G., $2003: 113$. 
Es importante mencionar que a su vez la Ciudad Autónoma de Buenos Aires tiene un Ente Único Regulador de los servicios públicos, pero que no controla el servicio del agua a pesar de tratarse de un servicio público. El servicio del agua quedó excluido del mismo debido a que en la Ciudad de Buenos Aires ya existían entes que controlaban la gestión del servicio (ERAS y APLA), y de esta manera se evitaron superposiciones legales de las funciones de los organismos.

Esto resulta contradictorio con lo establecido por la normativa local vigente, en tanto que en el artículo 48 de la Ley 3295 sobre gestión de agua de la Ciudad
Autónoma de Buenos Aires de 2009 se establece lo siguiente:

Servicio de agua potable y desagües cloacales. El Poder Ejecutivo instrumentará las acciones necesarias para que la Ciudad Autónoma de Buenos Aires asuma el ejercicio pleno de sus facultades concedentes y de control para la prestación de los servicios de provisión de agua potable y desagües cloacales en el ámbito de su territorio (L.3295/2009 -Art. 48).

Hasta el momento, esto no se ha implementado ni reglamentado, contradiciendo el espíritu y el texto de dicha norma.

\section{Gráfico No 5}

Planificación hidrológica y supervisión de obras

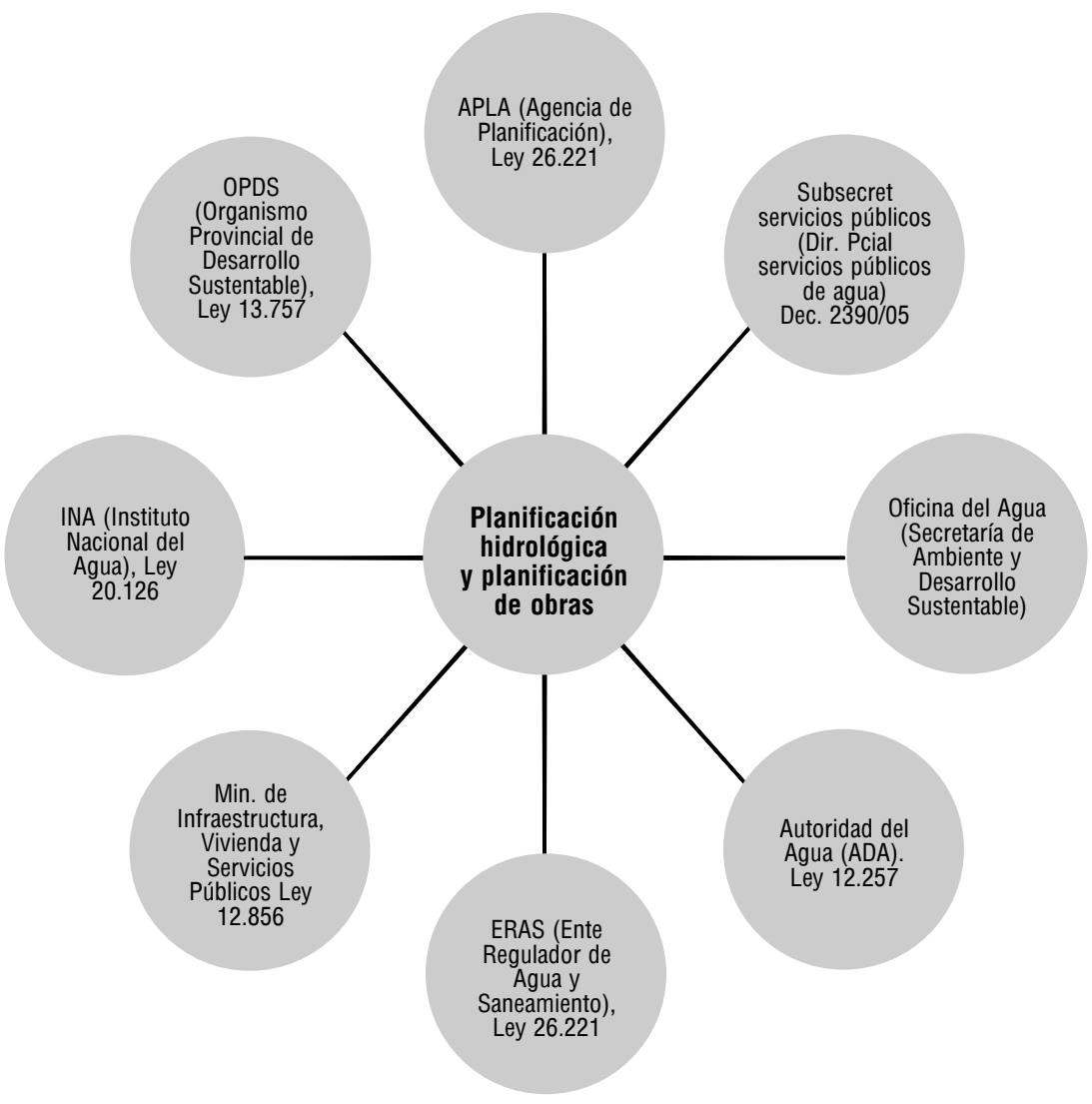

La planificación hidrológica y de las obras es otro aspecto fundamental vinculado con un adecuado servicio de agua, y en este ámbito concesional se encuentra regulado por ocho organismos, lo cual dificulta el eficiente control debido a que se requiere mayor coordinación y recursos financieros. 


\section{Gráfico $\mathrm{N}^{\circ} 6$}

Regulación del servicio del agua

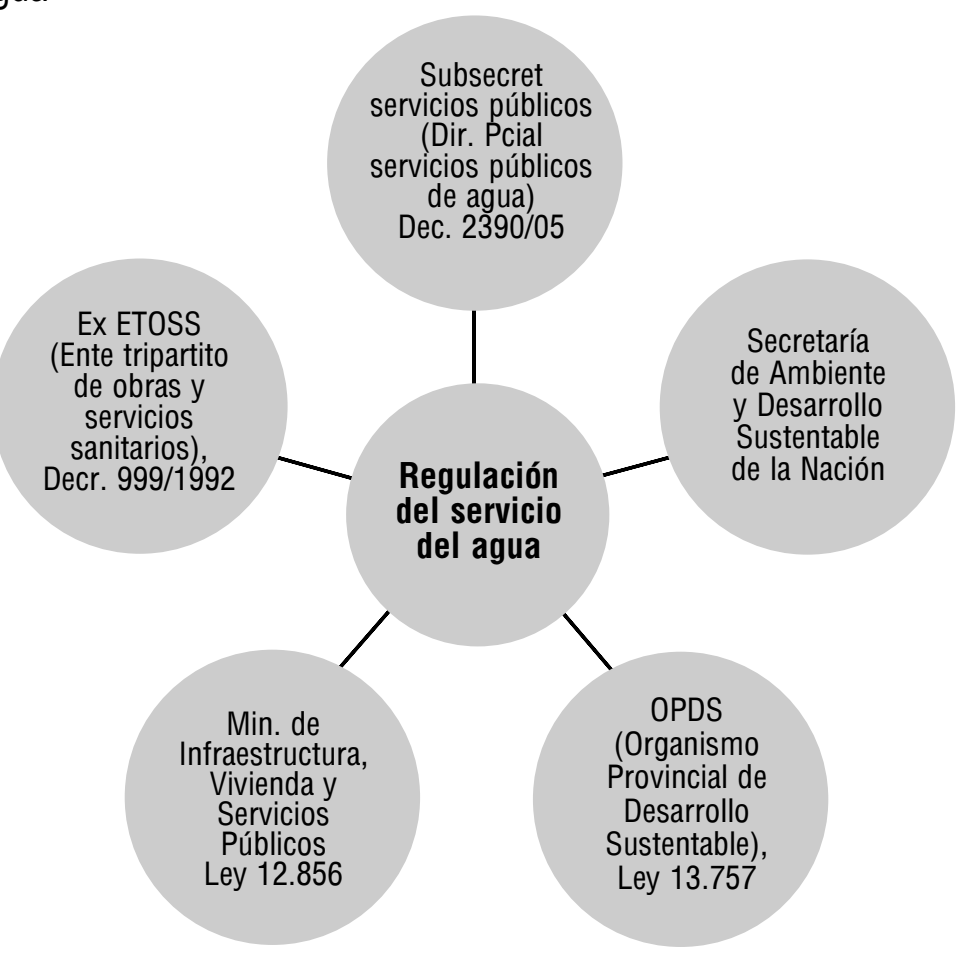

La función de regulación del servicio del agua es una de las más importantes. Esta define cuestiones fundamentales relacionadas con la gestión y provisión del recurso del agua, como son los derechos de los usuarios y de los consumidores.

\section{Gráfico $\mathrm{N}^{0} 7$}

Administración de la información

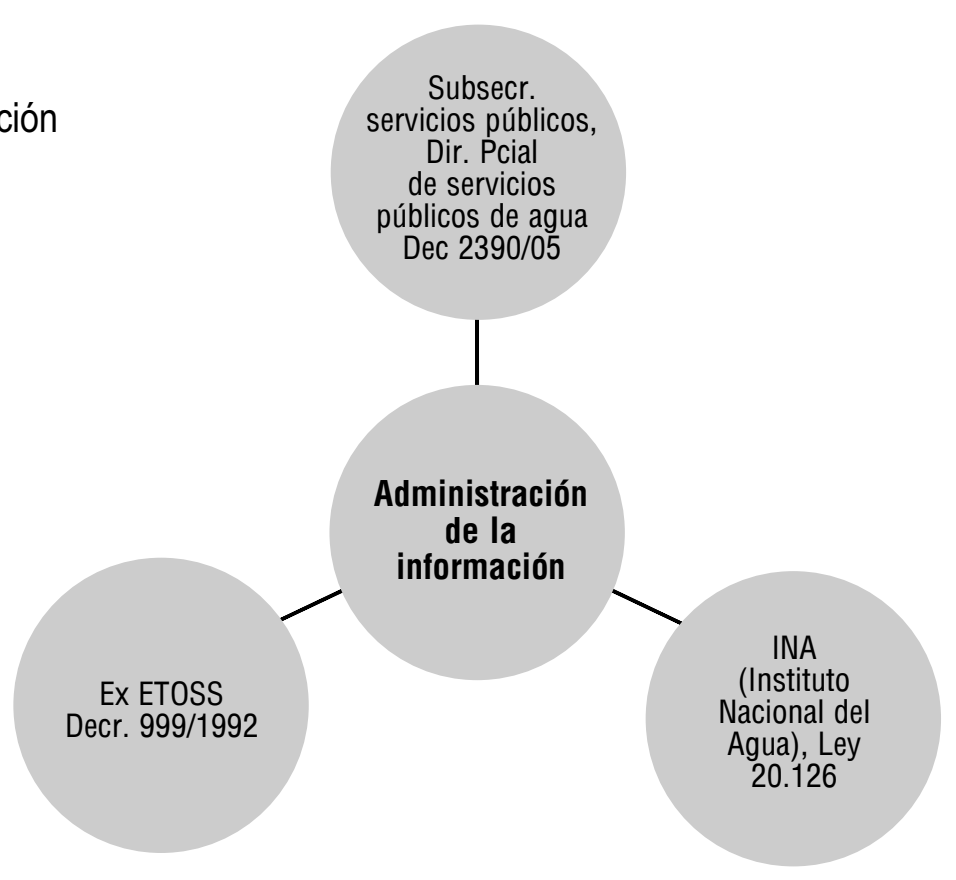

\section{0}


Debe destacarse que las funciones de los organismos descriptas en el cuadro anterior, representa uno de los aspectos que serán analizados más adelante en el capítulo destinado al derecho de acceso a la información. De todas maneras, debe mencionarse que son escasos los organismos que se dedican a la administración de la información vinculada al servicio del agua, el cual es de tanta importancia para la población.

\section{Gráfico $\mathrm{N}^{0} 8$}

Ejecución de programas de educación y política ambiental

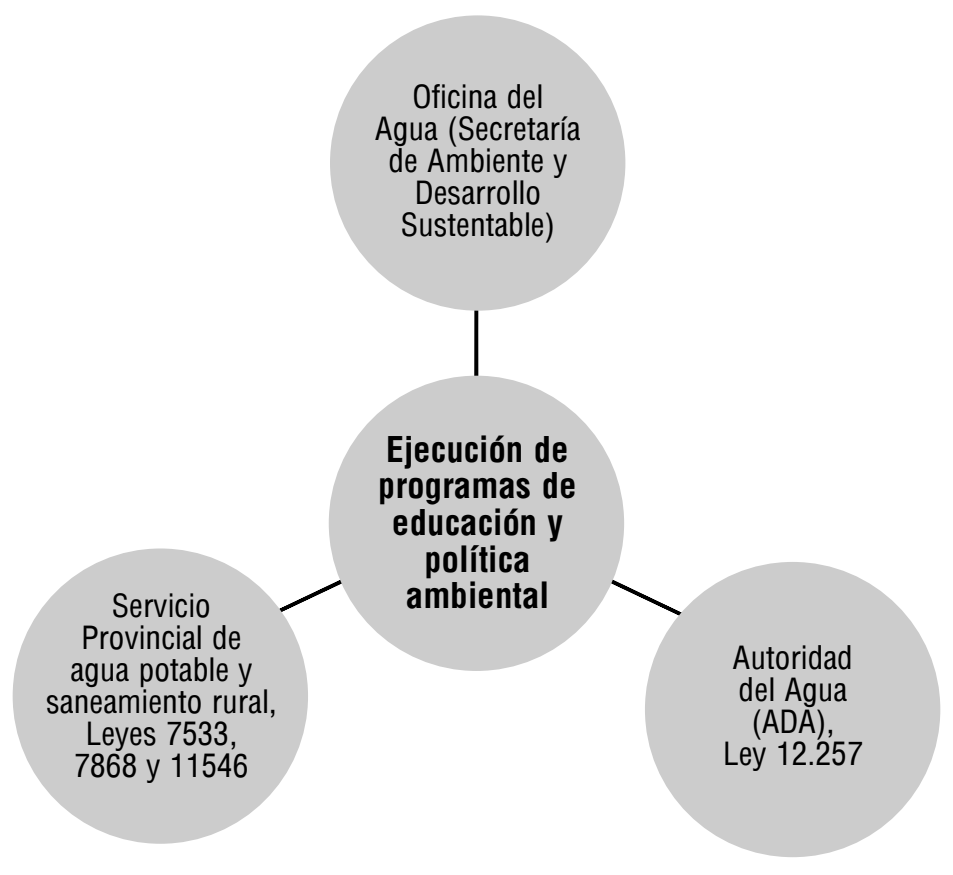

El derecho de acceso a la educación ambiental se vincula directamente con los de acceso a la información y con el de la defensa de los usuarios y consumidores. El mismo es implementado por tres organismos en la Provincia de Buenos Aires, lo que representa una situación satisfactoria ya que existe la obligación legal de invertir recursos públicos en dicha temática.

Una de las cuestiones fundamentales que surgió de los gráficos anteriores y que actualmente no se tienen en cuenta es la armonización y/o clarificación de las responsabilidades de los diferentes organismos, y la firma de acuerdos de cooperación entre los mismos (Rouse, 2007). Es evidente que aquí existe una inadecuada división legal de las funciones y de las responsabilidades, correspondientes a la mayoría de los organismos vinculados con el servicio de agua. Esto sin duda dificulta las tareas de coordinación, y además suma costos adicionales que deberán ser solventados por la Administración Pública.

Asimismo, luego de analizarse todas las obligaciones que surgen de la normativa de estos organismos y la información suministrada en las redes conceptuales, debe destacarse que existen áreas de solapamiento para el ámbito de la Provincia de Buenos Aires, en donde se generan situaciones dudosas respecto a qué organismo acudir ya que varios poseen la misma facultad para el mismo ámbito territorial. 
Debe tenerse en cuenta que la situación se agrava aún más, ya que la concesión de Aguas Argentinas y de Aysa abarcan a la Ciudad Autónoma de Buenos Aires y a la Provincia de Buenos Aires. Esto se fundamenta en que debido al escaso desarrollo normativo en la Ciudad de Buenos Aires en relación con el servicio de agua, se configuren vacíos legales como consecuencia del respeto a su autonomía constitucional.

A partir del análisis de la normativa vinculada con los organismos del servicio de agua, también han surgido otras cuestiones que son muy importantes.

En primer lugar, los entes de control son los que se ocupan en la práctica de preservar el recurso del agua, cuando en realidad sería mucho más beneficioso que esta tarea la desarrolle la autoridad u organismo provincial que posee competencia ambiental.

En el caso de Aysa se volvió a la misma estructura de control que se tenía en la empresa Obras Sanitarias de la Nación, en donde las funciones de prestación y de control de la empresa las realizaba el mismo ente. Esto es opuesto a lo que ocurre en Gran Bretaña, que posee una reconocida tradición institucional vinculada con el servicio de provisión del agua.

El caso de la "Inspección del Agua Potable" (Drinking Water Inspectorate) resulta ser ejemplificativo, ya que es uno de los tres entes reguladores que existen para el control de este servicio en el Reino Unido y en Gales. El mismo fue creado en 1990 y su función principal es la de asegurar una adecuada calidad del agua para consumo humano (Rouse, 2007, p.76). Se encuentra ubicado dentro del Departamento de Ambiente, Alimentos y Asuntos Rurales, al haberse tenido en cuenta que la aplicación del Derecho Ambiental era fundamental en la mejora de la calidad del servicio (cumpliendo los lineamientos de la Directiva europea 98/83/EC relativa a la calidad de las aguas), y además asesora sobre toda las normas vinculadas. Dicho organismo se encuentra dividido en tres partes:

Operaciones: Realizan auditorías técnicas en empresas.

Normativa: Controlan tarifas y el cumplimiento legal.

Ciencia y estrategia: Realiza tareas de planificación e investigación.

Además, este organismo también es responsable de lo siguiente:

- Examinar de forma independiente las actividades de las empresas que proveen agua a los consumidores.

- Trabajar conjuntamente con otros actores sociales para mejorar la calidad del agua para consumo humano, y para asegurar la calidad de la misma.

- Investigar sobre cuestiones vinculadas a la calidad y publicar información sobre agua potable. $^{8}$

Esta clase de organismo que posee funciones multisectoriales e integrales (que involucra al ámbito ambiental, legal, de investigación, y técnico), no existe en este ámbito de investigación, en tanto que el mismo debería ser incorporado a la Secretaría de Ambiente y Desarrollo Sustentable de la Nación.

\section{CONCLUSIONES}

El aspecto más problemático que se ha analizado en el presente artículo, se vincula con el funcionamiento de los organismos de control, a través del ejercicio de su poder de policía. Se afirma que la existencia de un alto nivel de cumplimiento legal de las empresas de agua, va a depender directamente de los siguientes ámbitos:

a) Funcionamiento de los organismos de control.

b) La calidad de la normativa vigente. 
Se ha detectado que actualmente el Estado se encuentra controlándose a sí mismo, ya que las dos empresas proveedoras de agua poseen mayoritariamente capitales públicos.

El funcionamiento insatisfactorio de los órganos de control del servicio es un problema que se repite en diversos países del mundo, pero que tiene una posible explicación en la existencia de una fragmentación de las agencias gubernamentales. ${ }^{9}$

La tendencia evidenciada en Buenos Aires y en Santa Fe es la del aislamiento de los sistemas de control, cuando la tendencia internacional manifestada por autores como Rouse es la contraria, ya que recomiendan integrar a los mismos para que sean más eficientes. En contraste con lo anterior, Rouse definió qué es lo que considera que es un órgano regulatorio "independiente" detallando las facultades que le caben: i). Fijar tarifas, ii). Imponer sanciones; iii). Aplicación normativa; iv). Comunicarse con la prensa, y, v). Informar sobre cualquier aspecto vinculado a las funciones regulatorias sin tener que dirigirse antes a nadie más (Rouse, 2007:29). Ninguno de los órganos fragmentados que se han analizado para los casos de Buenos Aires y Santa Fe, realizan conjuntamente dichasfunciones.

Otra de las cuestiones fundamentales detectadas en esta área es la dificultad legal vinculada con adaptar la normativa del ámbito nacional y provincial para la aplicación municipal.

A pesar de que la actual tendencia mundial va dirigida hacia la descentralización de la provisión de los servicios del agua, en el ordenamiento institucional de Argentina la autonomía municipal (entendida como la posibilidad de las sociedades locales de gobernarse por sí mismas sin injerencias de otras esferas del Estado), no constituye un concepto absoluto sino que presenta diferentes grados y tipos.
Además, se ha detectado una falta de claridad en las funciones de los organismos de control. Institucionalmente las funciones de los organismos públicos también son la causa de numerosas interferencias jurisdiccionales para la gestión coordinada del agua.

En virtud de lo anterior, cabe resaltar lo expresado por Dourojeanni (2002) quien afirma que "los cruces de funciones institucionales pueden resultar más conflictivos que los causados por la superposición de gobiernos con límites político administrativos y con límites naturales" (p.94). Una de las posibles justificaciones para la existencia de esta situación es que se han complejizado los actores vinculados con la gestión del recurso del agua, y que la normativa no haido evolucionando en forma paralela.

Además, a lo largo de la investigación se ha detectado que existe una superposición de organismos competentes, los cuales dificultan aún más el control. En este caso resulta fundamental "establecer con claridad la asignación de funciones y responsabilidades entre los diferentes organismos y jurisdicciones, de modo de asegurar el derecho de los habitantes a exigir información, detectar incumplimientos, efectuar denuncias y exigir remedios en casos de violaciones" (Centro de Estudios Legales y Sociales, 2009, p.9).

El mismo criterio fue utilizado para el caso del ámbito judicial en la Provincia de Buenos Aires, en donde el mismo se encuentra dividido en Departamentos que no coinciden con los Partidos ni los Municipios. Un ejemplo de esto podría graficarse en el caso del Poder Judicial de Mercedes que en su alzada las causas se remiten a la Plata, a pesar de que en muchos casos los jueces no conocen la realidad de la temática que deben resolver ya que no tienen contacto con la región ni con sus características particulares. 


\section{REFERENCIAS BIBLIOGRÁFICAS}

- Budds, J.; Mcgranahan, G. (2003). "Are the debates on water privatization missing the point? Experiences from Africa, Asia and Latin America", Environment and Urbanization, Vol. 15, $\mathrm{N}^{\circ} 2$, SAGE.

- Centro de Estudios Legales y Sociales (CELS), Asociación Civil por la Igualdad y la Justicia $(\mathrm{AClJ})$, Center on Housing and Rights and Eviction (COHRE) (2009). El acceso a agua segura en el área metropolitana de Buenos Aires. Una obligación impostergable, Programa Servicios Públicos, Buenos Aires.

- Dourojeanni, A. (2002). "Experiencias en la formación de organismos de cuenca en Iberoamérica", El Derecho de aguas en Iberoamérica y España: cambio y modernización en el inicio del tercer milenio, Tomo II, Madrid, España, Editorial Civitas.

- Ehrhardt, D.; Groom, E.; Halpern, J., O'Connor, S. (2007). "Economic regulation or urban water and sanitation services: some practical lessons", Water Sector Board Discussion Paper Series, $\mathrm{N}^{\circ} 9$, The World Bank Group.

- Fernández, D, Jouravlev, A, Lentini, E., Yurquina, A. (2009). "Contabilidad regulatoria, sustentabilidad financiera y gestión mancomunada: temas relevantes en servicios de agua y saneamiento", División de Recursos Naturales e Infraestructura, Comisión Económica para América Latina y el Caribe.
- Minaverry, Clara (2014), Tesis doctoral: El Derecho como herramienta para la erradicación de escenarios propicios para la corrupción en el servicio del agua, Facultad de Derecho, Universidad de Buenos Aires.

- Mutuberría Lazarini, V. (2007). Los servicios públicos urbanos como medios colectivos para la producción y reproducción de la vida de los sujetos en sociedad desde la perspectiva de la economía social. Análisis de experiencias de gestión colectiva en el Gran Buenos Aires, Tesis de Maestría en Economía Social, $2^{\circ}$ edición (2005-2007), Universidad Nacional General Sarmiento, Argentina, p. 25.

- Rouse, M. (2007). Institutional Governance and Regulation of Water Services, Londres, Reino Unido, International Water Association (IWA), ISBN: 1843391341, pp. 29, 25, 53, 76, 178.

- Solanes, M. (2008). "Common Regulatory Principles and Regulation of Water and Sanitation", International Institute for Sustainable Development, 2009.

- Transparency International, Informe global de la corrupción 2008. Corrupción en el sector del agua.

- United Nations Development Programme (Undp) (2011). Informe de lucha contra la corrupción en el sector del agua, Métodos, herramientas y buenas prácticas, Nueva York. 